\title{
Sporadic hemiplegic migraine: report of a case with clinical and radiological features
}

\author{
Rohit Bhatia $\cdot$ Soaham Desai $\cdot$ Manjari Tripathi $\cdot$ \\ Ajay Garg · M. V. Padma · Kameshwar Prasad · \\ Mamta B. Singh
}

Received: 12 May 2008/Accepted: 18 August 2008/Published online: 23 September 2008

(C) Springer-Verlag 2008

\begin{abstract}
A case of visual hallucination, headache and left hemiparesis is reported. The patient had a history of recurrent attacks of similar semiology for the previous 15 years. MRI brain revealed a cortical hyperintensity on T2W, FLAIR and diffusion weighted imaging (DWI) in the right cerebral hemisphere with a normal ADC (apparent diffusion coefficient) map and MR angiogram. Detailed workup for MELAS was negative. A diagnosis of sporadic hemiplegic migraine was made and he was managed conservatively. He made a gradual complete recovery over 2 weeks. He was discharged on flunarizine for prophylaxis and has remained asymptomatic over the ensuing 4 months. This interesting condition is reviewed and discussed herein.
\end{abstract}

Keywords Hemiplegic migraine $\cdot$ Headache $\cdot$ Migraine . Hemiplegia · Flunarizine

\section{Introduction}

Sporadic hemiplegic migraine is a rare subtype of migraine headache with aura defined as migraine attacks associated with some degree of motor weakness/hemiparesis during the aura phase and where no first- or second-degree relative (parent, sibling or child) has identical attacks [1]. The earliest description of this syndrome has been by Whitty

R. Bhatia $(\bowtie) \cdot$ S. Desai - M. Tripathi .

M. V. Padma · K. Prasad · M. B. Singh

Department of Neurology, All India Institute of Medical

Sciences, New Delhi 110029, India

e-mail: rohitbhatia71@yahoo.com

A. Garg

Department of Neuroradiology, All India Institute of Medical Sciences, New Delhi 110029, India et al. [2] in 1953. Bradshaw et al. in 1965 reported a series of 37 cases of hemiplegic migraine. However, not all patients in the series had recurrent episodes of hemiplegia [4]. Thomsen et al. [3] reported a population search based prevalence study in Denmark, which found 105 patients with sporadic hemiplegic migraine at a prevalence rate of $0.01 \%$. Based on their findings, sporadic hemiplegic migraine was assigned a separate subtype in the revised ICHD 2004, [1] with nearly 200 cases described so far, still there is sparse data regarding the radiologic findings, pathophysiology and treatment of acute attack as well as prevention of SHM. We describe here the clinical and radiologic findings in a patient with sporadic hemiplegic migraine.

\section{Case summary}

A 30-year-old male presented to us with a history of headache and left hemiparesis of 2-day duration. For about half an hour preceding the headache, he had experienced bright flashes of light in both his visual fields, which was followed by severe throbbing headache accompanied by photophobia, nausea and vomiting. Later, he became disoriented, confused and developed weakness of his left upper and lower limbs along with slurring of speech and difficulty in swallowing. There was no history of fever, trauma, seizure, or dehydration preceding the episode. In the past 16 years, he had had about 50 such episodes, starting at the age of 14 years. Most of these attacks were preceded by visualizing unformed flashes of light associated with black spots and a feeling of objects revolving and shimmering, making him feel as if he was on a giant wheel. This would be associated with a feeling of numbness involving the right arm and ascending up to his face in a peri-oral distribution. 
He also complained a sensation of objects crawling over his four limbs. This would be followed by intense headache and right sided weakness lasting 10-30 h. Twice in the preceding 4 years he had had experienced prolonged episodes of right upper and lower limb weakness associated with confusion and difficulty in speaking. In all other episodes, he would recover spontaneously and completely within 10-12 h. He was thought to have complex partial seizures and was being treated with carbamazepine in a dose of $900 \mathrm{mg} /$ day without any benefit. He had no history to suggest myoclonus, proximal muscle weakness, syncopal attacks, cardiac disease, coagulation disorder, connective tissue disease, hypertension, diabetes mellitus, smoking, alcoholism or any recreational drug abuse. There was no history of similar complaints in any of his family members as ascertained by personal interview and examination of all his first- and second-degree relatives. General physical examination and examination of chest, cardiovascular and abdomen was unremarkable. On nervous system examination, he was drowsy and disoriented to time, place and person. He had a bilateral asymmetric sixth nerve palsy (left $>$ right) and a left homonymous hemianopia. Fundus examination was normal. There was a left hemiparesis with a power of MRC grade 3/5 in his left upper and lower limbs and suggestion of a left hemineglect. His hemogram, coagulation profile, liver and renal functions, serum lipids, homocystiene, CPK, thyroid function, tests for prothrombotic state (protein $\mathrm{C}, \mathrm{S}$, anti-thrombin-3, factor $\mathrm{V}$ Leiden mutation, anti-phospholipid antibodies and lupus anticoagulant assay) and serum lactate were normal. His CT head revealed fullness of gyri on the right side involving the parieto-occipital region with no enhancement post contrast. MRI brain performed at $18 \mathrm{~h}$ of onset of attack revealed right cerebral hemisphere sulcal effacement and cortical prominence which was hyperintense on T2, FLAIR and DWI images with no abnormality on the ADC map bilaterally (Fig. 1a-e). Intracranial as well as extracranial MR angiography was normal. His echocardiogram was normal. His electroencephalogram revealed a generalized intermittent theta slowing without any epileptiform discharges. His CSF was acellular and protein, sugar and lactate levels were normal. His muscle biopsy did not reveal any abnormality. Genetic analysis for mitochondrial mutations MELAS A 3243 G, MELAS T 3271 C, MELAS 3291 C and MERRF A $8344 \mathrm{G}$ (for MELAS) was negative.

He was managed conservatively and made a gradual and complete recovery over the next 10 days. Flunarizine in a dose of $10 \mathrm{mg}$ per day was started for migraine prophylaxis and he did not have any further episodes of headaches or focal deficit over a 4-month follow-up. MRI study repeated after 4 weeks of symptom onset showed resolution of changes (Fig. 1f-h).

\section{Discussion}

Our patient had a history of visual and sensory symptoms accompanied by throbbing diffuse headaches and
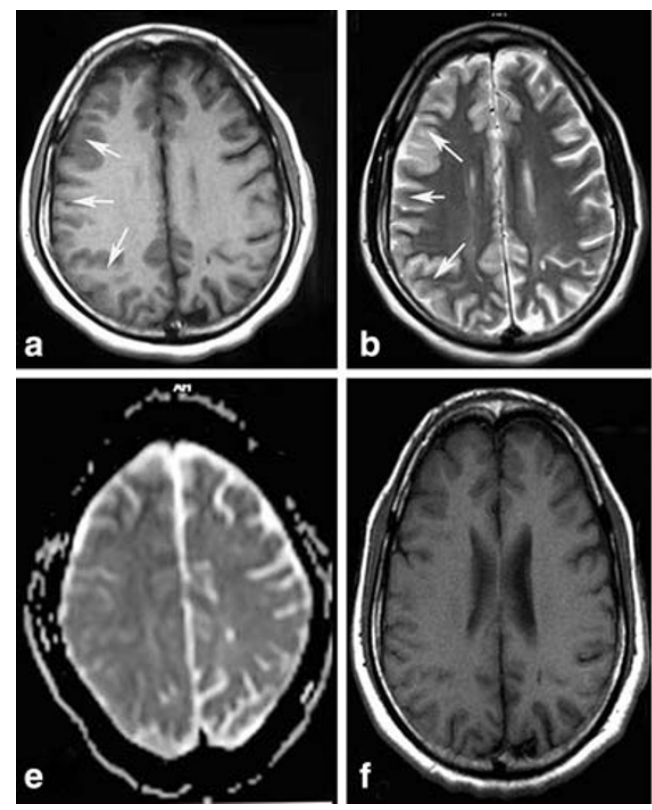

Fig 1 (a) T1-weighted axial MRI brain, reveals right cerebral hemispheric sulcal effacement and cortical thickening which is hyperintense on T2WI $(b)$, FLAIR $(c)$ and DWI $(d)$ images. There is

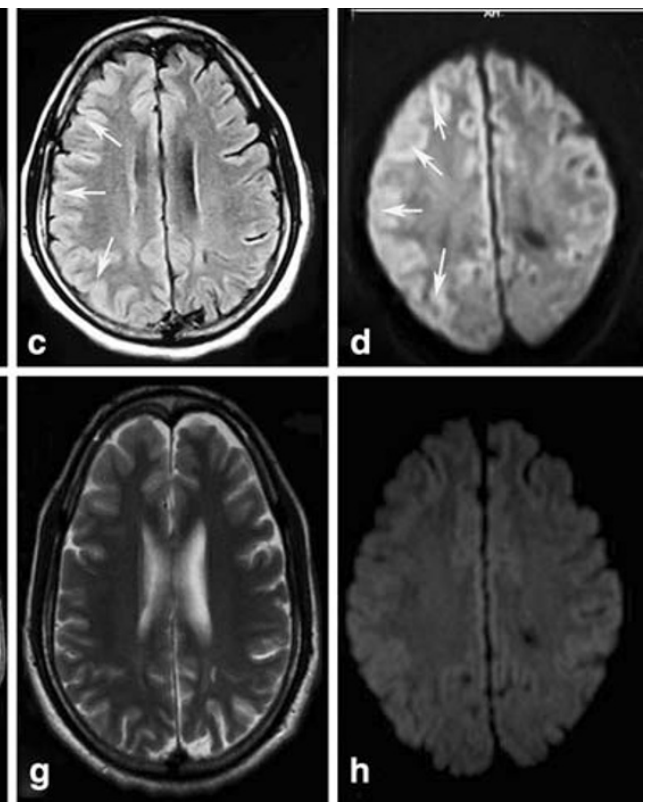

no evidence of altered signal on $\operatorname{ADC}$ map (e). Follow-up MR at 4 weeks shows resolution of changes $(f-h)$ 
associated hemiparesis. He did not have any migrainous headaches without aura separate from these episodes. None of his family members had experienced similar symptoms. The possible etiology of such a presentation could be any of the causes listed in Table 1. However, during epilepsy and TIA the symptoms generally do not have a gradual progression and are not associated with severe and prolonged post-ictal headache phase. In the present case, extensive work up as delineated above did not suggest the possibility of a stroke or epilepsy. MELAS presents with episodes of migrainous headaches and stroke-like focal deficits associated with increased lactate in serum and CSF during the attack. MRI brain often reveals T2 and FLAIR hyperintense lesions predominantly in the posterior cerebral cortex. Genetic testing for appropriate mitochondrial mutations and muscle biopsy with cytochrome oxidase staining and electron microscopy is generally diagnostic of this syndrome and was negative in our case. Pseudomigraine with lymphocytic pleocytosis and transient neurologic deficits is a very rare condition associated with single or multiple attacks of migraine-like headaches, weakness, sensory and visual auras with confusion and sometimes meningismus lasting minutes to hours. Typically, these patients have CSF lymphocytic pleocytosis $\left(10-760\right.$ cells $\left./ \mathrm{mm}^{3}\right)$ without elevation in protein or reduction in CSF glucose and have a normal brain imaging. However, in this syndrome, visual auras are very uncommon (12\%) as compared to sensory symptoms (78\%). Our patient had neither CSF abnormality nor any evidence of meningitis. The diagnosis of basilar migraine requires that there should be no motor weakness as a part of the aura. Although there was presence of other symptoms, the presence of hemiplegia would argue against this diagnosis in the present case. Migrainous infarction implies development of a stroke during the course of a migraine attack. It generally involves a large artery territory and corresponds to the side of the origin of migrainous auras in the

Table 1 Possible differential diagnosis of recurrent hemiplegia

\begin{tabular}{ll}
\hline 1 & Transient ischemic attacks and recurrent strokes \\
2 & Complex partial seizure with and without secondary \\
generalization (with post-ictal Todd's palsy) \\
3 & One of the migraine with aura subtypes: \\
(a) Hemiplegic migraine.(sporadic or familial) \\
(b) Basilar-type migraine \\
(c) Migrainous infarction (complication of migraine) \\
Mitochondrial encephalopathy with lactic acidosis \\
and stroke-like episodes (MELAS) syndrome \\
$5 \quad$ Pseudomigraine with lymphocytic pleocytosis \\
6 \\
with transient neurologic deficits
\end{tabular}

previous attacks. Its diagnosis requires the presence of prolonged hemiparesis associated with an MRI evidence of infarction. In the present case, the MRI revealed evidence of diffusion abnormality not involving any specific vascular territory with normal $\mathrm{ADC}$ and resolution at follow-up. Prolonged aura without infarction is defined as prolonged aura with symptoms and signs lasting more than 2 weeks without any radiologic evidence of infarction. Our patient fulfilled all criteria and best fits in the diagnosis of Migraine with aura and its subtype of sporadic hemiplegic migraine (ICHD criteria 1.2) [1, 3].

Most patients with sporadic hemiplegic migraine have 'typical' aura symptoms (visual, sensory and/or aphasic) associated with motor weakness during the acute attack $[3,4]$. About three-fourth of patients with sporadic hemiplegic migraine also fulfill the criteria for basilar-type migraine during the acute attack, but all such patients should be diagnosed as hemiplegic migraine rather than basilar-type migraine as per ICHD criteria $[1,3]$. The auras are more prolonged in sporadic hemiplegic migraine as compared to a typical migraine with aura [3]. The acute hemiplegic attack is always associated with severe headache while typical migraine with aura may occur without headache also $[1,3]$. Absence of infarction on imaging helps in differentiating it from migrainous infarction or stroke associated with migraine $[1,3]$.

The data on imaging abnormalities described in sporadic hemiplegic migraine as well as familial hemiplegic migraine is sparse. The findings described consist of either restricted diffusion, normal or increased diffusion based on DWI and ADC values involving a hemisphere opposite to the side of deficit [5-7] associated with normal T2W, T1W images, angiography and perfusion studies have shown hyperperfusion [5]. Magnetic resonance spectroscopy has suggested reduced myoinositol/creatinine ratio, reduced $\mathrm{N}$-acetylaspartate (NAA) on the affected side with normal choline/creatinine ratio. All these abnormalities have been found to be resolving gradually over 4-12 weeks [5]. These findings have been thought to represent a fully resolving diffusion metabolic abnormality without any vaso-occlusion, which could be due to the pathology at the cellular level. The restricted diffusion is correlated with cell swelling as a result of ATPase pump dysfunction [5,7]. Similarity of imaging findings in sporadic and familial hemiplegic migraine is suggestive of a possible common pathophysiology involving both the conditions which at present is thought to be due to a spreading depolarizing wave of neuroelectric or metabolic stimulus leading to prolonged neuronal depolarization. Interestingly, however, in the present case, the abnormality was in the form of hyperintense signal apparent on T2W and FLAIR imaging also, with hyperintense signal on DWI but no abnormalities on ADC map. These changes could possibly suggest vasogenic edema or a 
metabolic change as evidenced by complete reversibility on follow-up, both clinically and radiologically.

Treatment strategy at present is only on the basis of case reports of effective treatment with Flunarizine, Naloxane and Verapamil [8-10]. At present, SHM is treated on the same lines as familial hemiplegic migraine. Sympathomimetic drugs like ergotamine derivatives and triptans are avoided in acute attacks of hemiplegic migraine due to the fear of vasospasm leading to permanent sequelae. Similarly, beta-blockers are not used for prophylaxis in patients with hemiplegic or basilar-type migraine for the risk of prolonged aura or migrainous infarction. However, the most important part in the management of hemiplegic migraine, and especially in SHM, is that it is considered a diagnosis of exclusion, only after a thorough workup for other conditions has been carried out. At the same time, it is also important to keep SHM as a possibility during evaluation of patients with recurrent TIAs or epilepsy, especially if there is a history of hemiplegic episodes, which recover without any residual sequelae.

Conflict of interest None.

\section{References}

1. Headache Classification Committee of the International Headache Disorders (2004) 2nd edn. Cephalalgia 24:1-160

2. Whitty CWM (1953) Familial hemiplegic migraine. J Neurol Neurosurg Psychiatry 16:172-177

3. Thomsen LL, Olesen J (2004) Sporadic hemiplegic migraine. Cephalalgia 24:1016-1023

4. Bradshaw P, Parsons M (1965) Hemiplegic migraine A, clinical study. Q J Med 34:65-85

5. Jacob A, Mahavish K et al (2006) Imaging abnormalities in sporadic hemiplegic migraine on conventional MRI, diffusion and perfusion MRI and MRS. Cephalalgia 26(8):1004-1009

6. Butteriss DJ, Ramesh V et al (2003) Serial MRI in a case of familial hemiplegic migraine. Neuroradiology 45:300-303

7. Chabriat H, Vahedi K et al (2000) Decreased hemispheric water mobility in hemiplegic migraine related to mutation of CACNA1A gene. Neurology 54:510-512

8. Centonze V, Brucoli C, Macinagrossa G, Attolini E, Campanozzi F, Albano O (1983) Non-familial hemiplegic migraine responsive to naloxone. Cephalalgia 3:125-127

9. Yu W, Horowitz SH (2003) Treatment of sporadic hemiplegic migraine with calcium-channel blocker verapamil. Neurology 60:120-121

10. Tobita M, Hino M, Ichikawa N, Takase S, Ogawa A (1987) A case of hemiplegic migraine treated with flunarizine. Headache $27: 487-488$ 